\title{
Improved electrochemical analysis of neuropathy target esterase activity by a tyrosinase carbon paste electrode modified by 1 -methoxyphenazine methosulfate
}

\author{
L.G. Sokolovskaya ${ }^{1}$, L.V. Sigolaeva ${ }^{1}$, A.V. Eremenko ${ }^{1}$, I.V. Gachok ${ }^{1}$, G.F. Makhaeva ${ }^{2}$, \\ N.N. Strakhova ${ }^{2}$, V.V. Malygin ${ }^{2}$, R.J. Richardson ${ }^{3}$ \& I.N. Kurochkin ${ }^{1, *}$ \\ ${ }^{1}$ Faculty of Chemistry, Moscow State University, 119899, Moscow, Russia \\ ${ }^{2}$ Institute of Physiologically Active Compounds, Russian Academy of Sciences, 142432, Moscow Region, \\ Chernogolovka, Russia \\ ${ }^{3}$ Toxicology Program, University of Michigan, Ann Arbor, Michigan, 48109, USA \\ *Author for correspondence (Fax: +7-095-9394391; E-mail: ikur@genebee.msu.ru)
}

Received 29 October 2004; Revisions requested 12 November 2004; Revisions received 8 June 2005; Accepted 10 June 2005

Key words: biosensor, mediator, 1-methoxyphenazine methosulfate, neuropathy target esterase, tyrosinase

\begin{abstract}
A graphite-paste tyrosinase biosensor was improved by adding 1-methoxyphenazine methosulfate as a mediator. Mediator modification enhanced sensitivity to phenol 4-fold and long-term stability 3-fold. Phenol could be detected at $25 \mathrm{~nm}(\mathrm{~S} / \mathrm{N}=2)$ using an $\mathrm{Ag} / \mathrm{AgCl}$ reference electrode. The biosensor was used to measure the activity of a toxicologically significant enzyme, neuropathy target esterase (NTE), which yields phenol by hydrolysis of the substrate, phenyl valerate. Using the new biosensor, blood and brain NTE inhibition by organophosphorus (OP) compounds with different neuropathic potencies were well correlated $(r=0.990, n=7)$, supporting the use of blood NTE as a biochemical marker of exposure to neuropathic OP compounds.
\end{abstract}

\section{Introduction}

Tyrosinase biosensors can detect phenol electrochemically at sub-nanomolar levels (Kotte et al. 1995, Shan et al. 2002, Wang et al. 2002). Accordingly, such electrodes are widely used, mainly for detection of phenols as environmental pollutants. Phenolic biosensors can also monitor enzymatic processes that yield phenols.

Tyrosinase biosensors have been used for electrochemical analysis of a toxicologically significant enzyme, neuropathy target esterase (NTE) (Sigolaeva et al. 1999, 2001, Makhaeva et al. 2003). NTE hydrolyzes the substrate, phenyl valerate, to produce phenol. Inhibition of NTE by neuropathic organophosphorus (OP) compounds initiates OP compound-induced delayed neurotoxicity (OPIDN) (Johnson 1982,
Glynn 1999, Huggins \& Richardson 1999). Inhibition of brain NTE within hours of exposure to a neuropathic OP compound predicts the onset of OPIDN in adult hens 1-3 weeks later (Johnson 1982, 1990, Lotti 1986, Richardson 1995, 1998). Tyrosinase biosensors enable NTE to be assayed in whole blood, which cannot be done with the standard colorimetric method (Sigolaeva et al. 2001). The original tyrosinase carbon-paste biosensor was used to establish correlations of NTE inhibition in blood with that in lymphocytes and brain $24 \mathrm{~h}$ after dosing hens with a neuropathic OP compound, $O, O$-di1-propyl $O$-2,2-dichlorvinyl phosphate. These studies demonstrated that the biosensor NTE assay for whole blood could serve as a biomarker of exposure to neuropathic OP compounds (Makhaeva et al. 2003). 
The present report describes the preparation of a 1-methoxyphenazine methosulfate (MPMS)modified tyrosinase carbon-paste electrode for improved electrochemical analysis of NTE activity in blood for the enhancement of biomonitoring exposures to neuropathic OP compounds.

\section{Materials and methods}

\section{Chemicals}

Mushroom tyrosinase (monophenol monooxidase, EC 1.14.18.1), activity $3800 \mathrm{U} / \mathrm{mg}$ for Ltyrosine, graphite powder, phenol, MPMS, and Paraoxon ( $O, O$-diethyl-4-nitrophenyl phosphate) were purchased from Sigma. Phenyl valerate was from Oryza (Chelmsford, USA). $N, N^{\prime}$-di-2propylphosphorodiamido fluoridate (Mipafox), $O, O$-diethyl $O$-2,2-dichlorovinylphosphate, $O, O$ di-1-propyl $O-2,2$-dichlorovinylphosphate and the $O$-alkyl- $O$-methylchloroformimino phenylphosphonate compounds $\left[(\mathrm{RO}) \mathrm{C}_{6} \mathrm{H}_{5} \mathrm{P}(\mathrm{O}) \mathrm{ON}=\mathrm{CClCH}_{3}\right.$ $(\mathrm{R}=\mathrm{alkyl})]$ were synthesized and characterized in the Institute of Physiologically Active Compounds, Russian Academy of Sciences (RAS) (Russia). All other chemicals were of analytical grade and used without further purification. Aqueous solutions were prepared using deionized water.

\section{Preparation of tyrosinase electrode and assembly of biosensor}

The tyrosinase electrode was prepared as described below. Graphite/paraffin oil $(3.5: 1, \mathrm{w} / \mathrm{w})$ were mixed thoroughly and packed firmly into a plastic holder ( $2 \mathrm{~mm}$ diameter). Electrical contact was established by a $0.2-\mathrm{mm}$ copper wire inserted from the rear. The surface of the electrode was covered by the composite of tyrosinase, graphite, MPMS, and paraffin oil (2:5.6:1:2, by weight), and then the surface was polished. The electrodes were stored dry at $4{ }^{\circ} \mathrm{C}$ until use. In experiments with optimization of tyrosinase concentration in carbon composite, tyrosinase, graphite, MPMS, and paraffin oil were $[x:(7.6-x): 1: 2$, by weight]. The enzyme amount $(x)$ was varied from 0.2 to $2 \mathrm{mg}$. The components ratio of [2:(6.6-y):y:2, by weight] was used for optimization of MPMS con- centration. MPMS amount $(y)$ was in the range of $0.2-1.4 \mathrm{mg}$.

\section{Electrochemical measurements}

All measurements were performed with an IPC2000 potentiostat (Institute of Physical Chemistry, RAS). The amperometric current response was recorded at $-150 \mathrm{mV}$ vs. the $\mathrm{Ag} / \mathrm{AgCl}$ reference electrode in a flow-through electrochemical cell after an injection of the samples into a flow of sodium phosphate buffer with $0.1 \mathrm{M} \mathrm{NaCl}$, via an injector with a $50 \mu \mathrm{l}$ sample loop (Valve V-7, Pharmacia, Sweden) at a flow rate of $0.25 \mathrm{ml} / \mathrm{min}$. Each measurement was made in triplicate.

The cyclic voltammograms of the electrodes were performed in a $1 \mathrm{ml}$ electrochemical cell with stirring in the $o$-quinone redox potential range. In all cases, a third cyclic voltammogram was used for calculations.

\section{Hen brain NTE assay}

A lyophilized hen brain membrane fraction consisting of combined mitochondrial/synaptosomal and microsomal pellets $\left(\mathrm{P}_{2}+\mathrm{P}_{3}\right)$ (Richardson et al. 1979) preinhibited with paraoxon $(40 \mu \mathrm{M}$ at $25^{\circ} \mathrm{C}$ for $45 \mathrm{~min}$ ) was used as a source of NTE. It was prepared as described (Makhaeva \& Malygin 1999) and stored in sealed ampoules. Before use, ampoule contents were suspended with a Potter homogenizer in $5 \mathrm{ml}$ of working buffer $(50 \mathrm{~mm}$ Tris/ $\mathrm{HCl}, 0.2 \mathrm{~mm}$ EDTA, $\mathrm{pH} 8.0$ at $25^{\circ} \mathrm{C}$ ).

NTE activity was determined colorimetrically according to the differential inhibition method of Johnson (1977) as modified by Kayyali et al. (1991) at $492 \mathrm{~nm}$ using a Benchmark Plus Microplate Reader (Bio-Rad, USA). Each measurement was made in triplicate. Protein was determined by the microbiuret method with bovine serum albumin as a reference standard.

\section{Whole blood NTE assay}

Whole hen blood stabilized by citrate/EDTA was diluted 10 -fold with working buffer and homogenized in a Potter glass/glass homogenizer before NTE assay. The differential inhibition method of Johnson (1977) with an electrochemical endpoint 
was used as described in (Makhaeva et al. 2003). Each measurement was made in triplicate.

Protein was determined using a Coomassie protein assay kit from Sigma (USA) with bovine serum albumin as a reference standard.

\section{NTE $\mathrm{I}_{50}$ determinations}

The $I_{50}$ (the concentration that inhibits $50 \%$ of the enzyme activity under defined preincubation conditions) for inhibitors against NTE was measured by preincubating a sample of enzyme with 10-12 different concentrations of the OP compound for $20 \mathrm{~min}$ at $37^{\circ} \mathrm{C}$, in working buffer. Residual NTE activity was then determined either using the colorimetric (brain NTE) or the amperometric (blood NTE, the MPMS-modified tyrosinase carbon-paste electrode) method. Each measurement was made in triplicate (colorimetry) or in duplicate (amperometry). $I_{50}$ values were calculated using Origin 6.1 software (Northampton, MA, USA). Every value represents the mean \pm SEM from three independent experiments.

\section{Results and discussion}

\section{Mediator-modified tyrosinase carbon paste electrode}

The tyrosinase carbon paste electrode involves oxidation of phenol via catechol into $o$-quinone by the tyrosinase. Then $o$-quinone is electrochemically reduced to catechol directly at the electrode when the required potential is applied. However, regeneration via mediator gives an increased sensitivity to phenol compared to the direct electroreduction of $o$-quinone (Figure 1).

We used MPMS as a light-stable mediator for the tyrosinase sensor modification. Various aspects concerning the electrode construction and operation have been studied and optimized as detailed below.

\section{Cyclic voltammetry}

Figure 2 shows the cyclic voltammograms of the mediator-modified tyrosinase electrode in $10 \mu \mathrm{M}$ phenol solution at various potential scan rates in the range between 2 and $40 \mathrm{mV} / \mathrm{s}$.

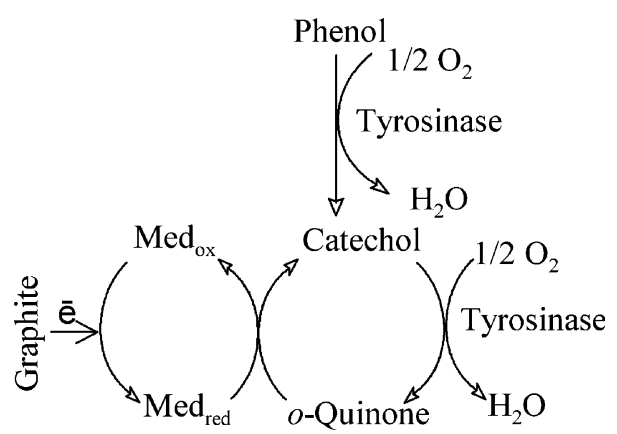

Fig. 1. Principle of amperometric phenol detection by 1-methoxyphenazine methosulfate (MPMS)-modified tyrosinase carbon-paste electrode.

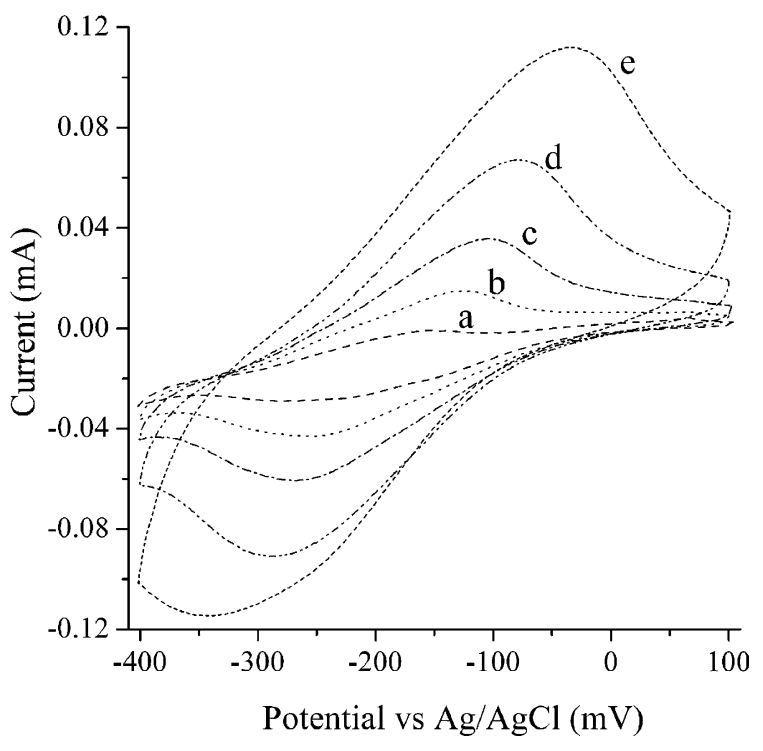

Fig. 2. Cyclic voltammograms of the 1-methoxyphenazine methosulfate (MPMS)-modified electrode at various potential scan rates. Conditions: tyrosinase and MPMS contents in carbon paste were $2 \mathrm{mg}$ and $1 \mathrm{mg}$, respectively; $0.05 \mathrm{~m}$ phosphate buffer solution with $0.1 \mathrm{M} \mathrm{NaCl} ; \mathrm{pH} 7.0 ; 10 \mu \mathrm{M}$ phenol. The potential scan rates were (a) 2, (b) 5, (c) 10, (d) 20 and (e) $40 \mathrm{mV} / \mathrm{s}$ vs. $\mathrm{Ag} / \mathrm{AgCl}$.

Both the oxidation and reduction potential values depended upon the scan rate. In addition, the cathodic and anodic peak currents coincided, and the nonlinearity depended upon the square root of the potential scan rate (data not shown). Thus, the redox system considered represents quasi-reversible character, and the polymeric quinoid products that can irreversibly bind and poison the electrode surface apparently do not readily form. 


\section{Optimization of carbon paste composite}

The influence of tyrosinase and mediator concentrations in the carbon composite on analytical properties of the modified electrodes was studied by screening electrode response to the addition of standard phenol solution. The electrodes were prepared as described above. The maximum response was achieved with $2 \mathrm{mg}$ tyrosinase. Further increasing enzyme content resulted in deterioration of the physical properties of the carbon composite resulting in peeling off the active paste layer from the electrode surface. The effect of MPMS concentration in the carbon composite on the electrode response was assessed between 0.2 and $1.4 \mathrm{mg}$ MPMS. The electrodes were prepared as described above. The electrode response was found to increase to a plateau at $0.6 \mathrm{mg}$ of the mediator content. Thus, a signal gain of more than four times was achieved in comparison with the unmodified electrode.

\section{Optimization of measuring conditions}

The effect of operating potential on the mediator-modified electrode response was examined in the range between -25 and $-250 \mathrm{mV}$ vs. $\mathrm{Ag} /$ $\mathrm{AgCl}$ in comparison with the unmodified electrode. As seen in Figure 3, the highest signals are obtained at potential values between -175 and $-200 \mathrm{mV}$ for both electrode types. Nevertheless, the recommended optimal operating potential is $-150 \mathrm{mV}$, because the background current significantly decreases in that case, while the electrode responses remain high. Furthermore, the relatively low operating potential decreases the influence of interfering electrochemical reactions on selectivity and sensitivity of the assay.

The influence of $\mathrm{pH}$ was studied from 4.5 to 9.5. The addition of the mediator to the carbon paste composite did not shift the $\mathrm{pH}$ optimum of immobilized tyrosinase (Duckworth \& Coleman 1970), which remained at $\mathrm{pH}$ 7.0.

Figure 4 shows the influence of ionic strength on the character of response of both the mediator-modified and unmodified electrodes to $10 \mu \mathrm{M}$ phenol. The response of the unmodified electrode increased with rising $\mathrm{NaCl}$ concentration up to $0.05 \mathrm{M}$. Further increases in ionic strength of the buffer solution had no effect on electrode response. The mediator-modified electrode

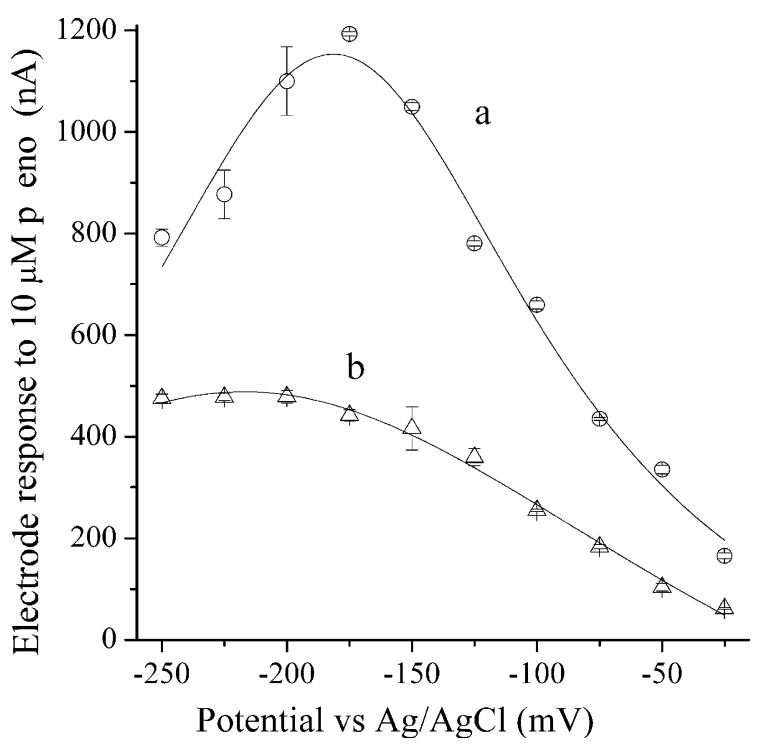

Fig. 3. Effect of the operating potential on the response current of 1-methoxyphenazine methosulfate (MPMS)-modified (a) and unmodified (b) electrodes. Conditions: $0.05 \mathrm{M}$ phosphate buffer solution with $0.1 \mathrm{M} \mathrm{NaCl}$; pH 7.0; $10 \mu \mathrm{M}$ phenol.

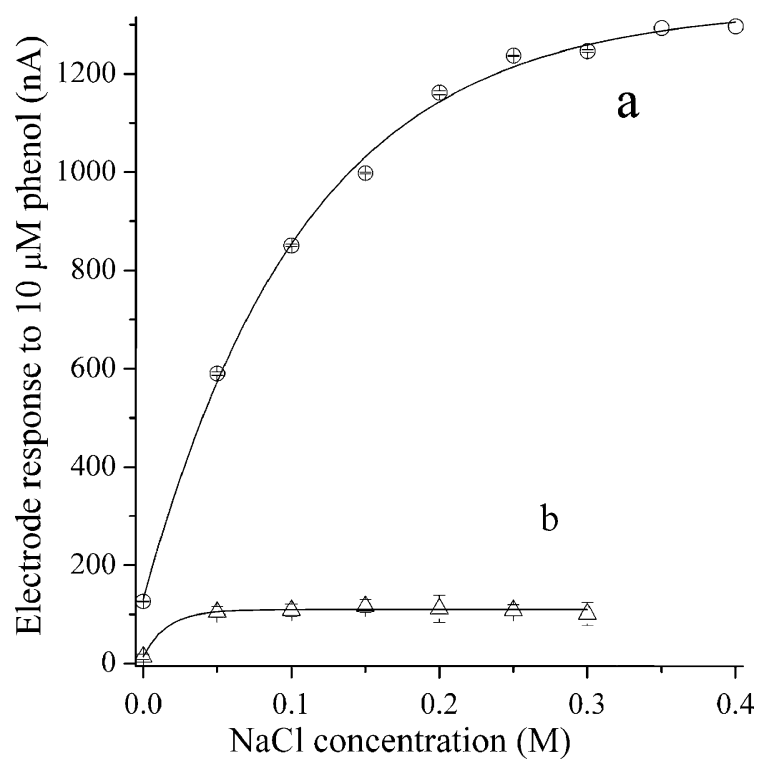

Fig. 4. Influence of $\mathrm{NaCl}$ concentration on the response of 1methoxyphenazine methosulfate (MPMS)-modified (a) and unmodified (b) electrodes. Conditions: $0.05 \mathrm{M}$ phosphate buffer solution containing ( 0 to $0.35 \mathrm{M}) \mathrm{NaCl}, \mathrm{pH} 7.0 ; 10 \mu \mathrm{M}$ phenol.

response increased with rising $\mathrm{NaCl}$ concentration up to $0.35 \mathrm{M}$, and exhibited about a two-fold increase in electrode response in a comparison 
with standard conditions of measurements at $0.1 \mathrm{M} \mathrm{NaCl}$.

\section{Analytical characteristics of MPMS-modified tyrosinase carbon paste electrodes}

The dependence of the MPMS-modified electrode responses on a wide range of phenol concentration is shown in Figure 5. The response was linear up to $50 \mu \mathrm{m}$. The detection limit of $25 \mathrm{~nm}(\mathrm{~S} /$ $\mathrm{N}=2$ ) was reached for the mediator-modified electrode, while the detection limit of the unmodified electrode was $100 \mathrm{~nm}$.

The operational stability of MPMS-modified electrodes was examined by serial measurements of $100 \mu \mathrm{M}$ phenol. After 80 measurements, the electrode retained its initial activity with a coefficient of variation of less than $3 \%$.

The results on functional stability are shown in Figure 6. Data were obtained for electrodes used in the flow-injection system at room temperature during the day and stored overnight at $4{ }^{\circ} \mathrm{C}$ in the working buffer solution. The unmodified electrode lost about $80 \%$ of its initial activity after 9 days. In contrast, the response of the MPMS-modified electrode decreased $23 \%$ after 9 days and $37 \%$ after 15 days. In the dry state at $4{ }^{\circ} \mathrm{C}$, the electrodes keep their initial activity for more than 12 months.

The main analytical characteristics of MPMSmodified electrodes are summarized in Table 1.

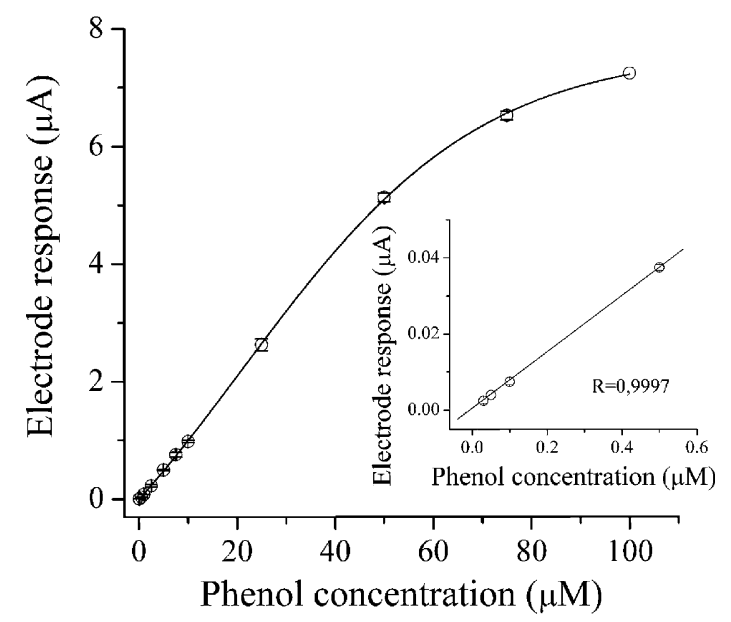

Fig. 5. Calibration curve of phenol at the methoxyphenazine methosulfate (MPMS)-modified electrode. Conditions: $0.05 \mathrm{M}$ phosphate buffer solution with $0.1 \mathrm{M} \mathrm{NaCl}$; $\mathrm{pH}$ 7.0; applied working potential $-150 \mathrm{mV}$ vs. $\mathrm{Ag} / \mathrm{AgCl}$.

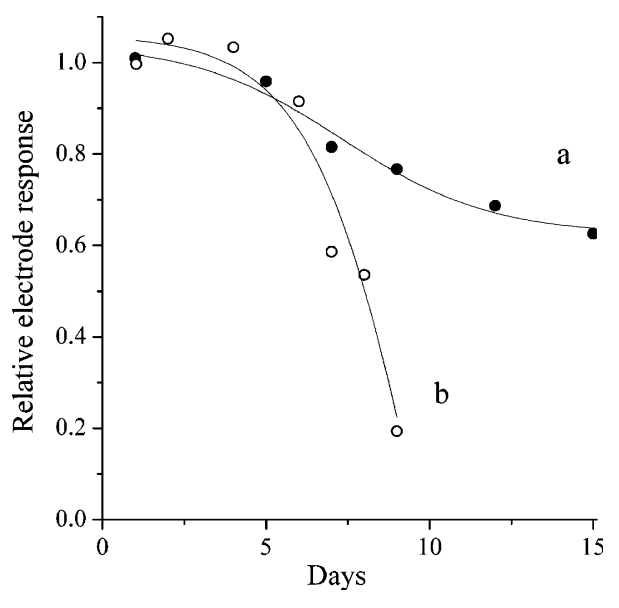

Fig. 6. Storage stability of the 1-methoxyphenazine methosulfate (MPMS)-modified (a) and unmodified (b) electrodes in $0.05 \mathrm{M}$ phosphate buffer solution with $0.1 \mathrm{M} \mathrm{NaCl} ; \mathrm{pH} 7.0$; $4{ }^{\circ} \mathrm{C}$. Relative electrode response values (ordinate axis) represent the electrode response obtained in a period from 1 to 15 days of storage against the initial electrode response obtained on the first experimental day.

Table 1. Characteristics of the phenol biosensor based on 1methoxyphenazine methosulfate (MPMS)-modified tyrosinase carbon paste electrode.

\begin{tabular}{ll}
\hline Analytical parameter & Value \\
\hline Linear range & $0.025-50 \mu \mathrm{M}$ \\
Detection limit of phenol (S/N=2) & $25 \mathrm{~nm}$ \\
Sample volume & $50 \mu \mathrm{l}$ \\
Time of analysis & $2 \mathrm{~min}$ \\
Work stability & $>60 \%$ during 21 days \\
Storage stability (dry) & $>12$ months \\
Coefficient of variation of & $25 \%$ \\
electrode preparation & \\
Coefficient of variation of 80 & $3 \%$ \\
consecutive phenol assays & \\
\hline
\end{tabular}

Whole blood NTE analysis as a biomarker of neuropathic OP compound exposure

We previously showed that the tyrosinase carbonpaste electrode remarkably improved the sensitivity of the NTE assay in comparison with a colorimetric method (Sigolaeva et al. 2001). Due to the high sensitivity of the biosensor method, the influence of interfering blood components (ascorbic acid, tyrosine, and others) was reduced by the high extent of sample dilution, thus allowing NTE to be detected selectively and with high sensitivity in whole blood, for which the usual colorimetric 
Table 2. The in vitro comparison of sensitivity of hen blood and brain NTE to OP compounds with different neuropathic potential.

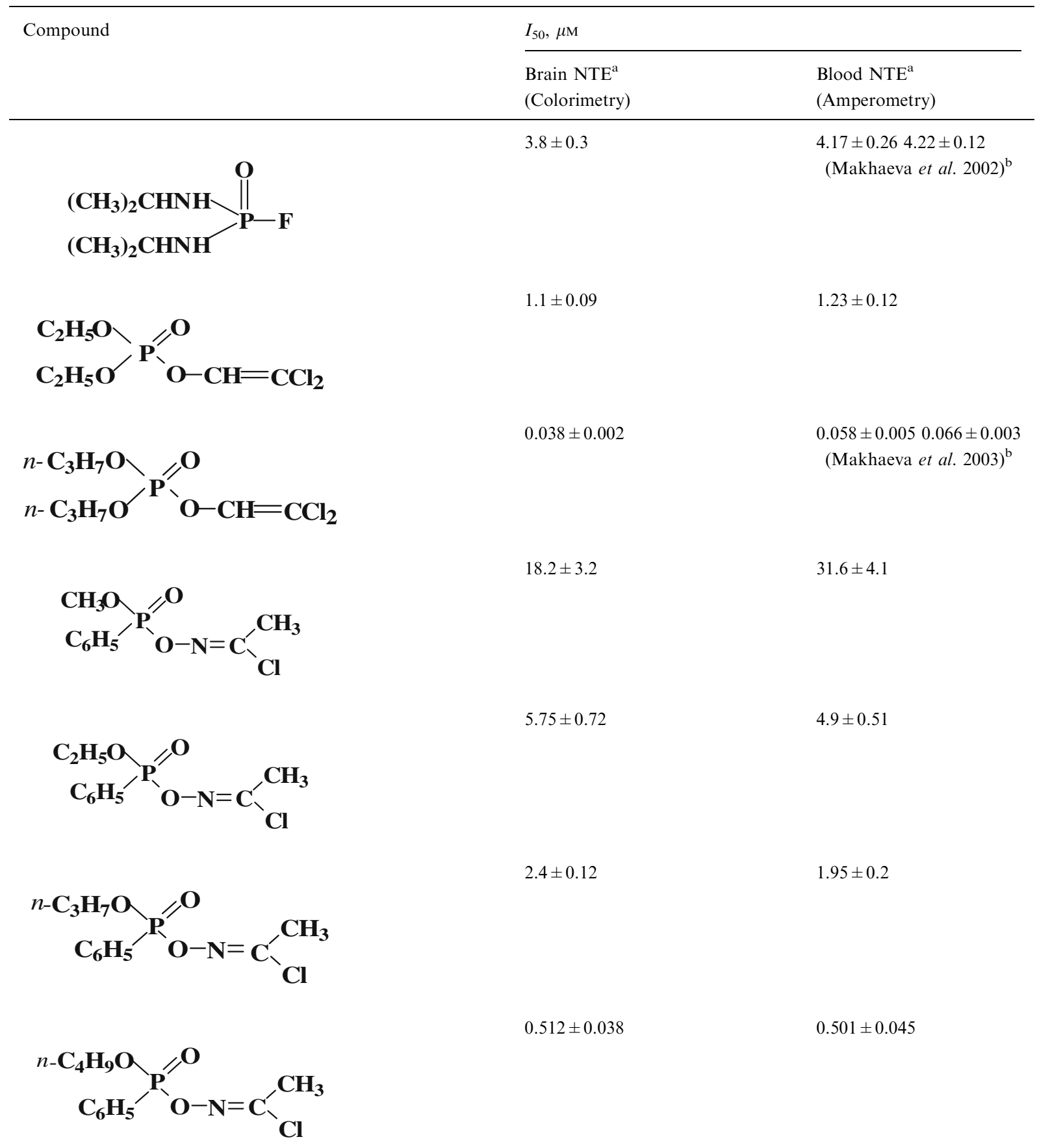

${ }^{\mathrm{a} C o n t r o l}$ NTE activities, $\mathrm{nmol} /(\min \times \mathrm{mg}$ protein), mean $\pm \mathrm{SEM}, n=3$. Lyophilized preparation of hen brain NTE $=41 \pm 2$, whole blood $=0.23 \pm 0.02$.

${ }^{\mathrm{b}} I_{50}$ was determined using the unmodified tyrosinase carbon-paste electrode. 
assay is impossible. Using the unmodified electrode, whole blood samples could be diluted 1:500 $(\mathrm{v} / \mathrm{v})$. Dilution of 1:1400 (v/v) was achieved with the MPMS-modified electrode.

Blood NTE activity measurements with the new MPMS-modified tyrosinase carbon-paste electrode were explored as a possible biochemical marker of neuropathic OP compound exposures. The biosensor assay was used to obtain $I_{50}$ values for inhibition of blood NTE activity in vitro with a number of standard neuropathic OP compounds. These include mipafox, $O, O$-diethyl- and $O, O$-di-1-propyl $O$-dichlorovinyl phosphates, as well as a series of $O$-alkylphenyl phosphonates of general formula $\mathrm{RO}\left(\mathrm{C}_{6} \mathrm{H}_{5}\right) \mathrm{P}(\mathrm{O}) \mathrm{ON}=\mathrm{CCH}_{3} \mathrm{Cl}$ where $\mathrm{R}=\mathrm{Me}$, Et, $n$-Pr, $n$-Bu. The neuropathic potential in the last series of compounds was shown to increase with R-group length (Makhaeva et al. 2001, Malygin et al. 2003). The standard colorimetric assay was used to determine the $I_{50}$ for these compounds against a preparation of hen brain NTE. The data presented in Table 2 show that all inhibitors exhibited a similar inhibitory potency in vitro toward NTE from hen brain and hen blood. NTE from both sources had the same sensitivity to variations in structure and neuropathic potential of OP inhibitors. A good correlation was found between the values of $\mathrm{p} I_{50}$ for blood and brain NTE inhibition by the studied compounds $(r=0.990, n=7)$. Furthermore, consistent agreement was obtained between two amperometric methods (MPMSmodified and unmodified electrodes) for $I_{50}$ determinations of mipafox and $O, O$-di-1-propyl $O$-dichlorovinyl phosphate against blood NTE. The data support the validity of measurements carried out with the new MPMS-modified tyrosinase carbon paste biosensor and demonstrate that blood NTE inhibition is a promising biomarker of exposure to neuropathic OP compounds.

\section{NTE Analyzer}

A pilot device (Analyzer) incorporating the new biosensor for NTE assays was developed. The Analyzer consists of an internal microprocessor, flow-through electrochemical cell supplied with working and reference electrodes, pump, sampler, vessels for working buffer and waste, liquid crystal display, and controls for manual operation. All functions are controlled and monitored by an external computer. The software provides measurement monitoring, and displays the measured signal in real-time format. Data processing is also possible.

The application of this device is expected to facilitate the rapid assessment of human exposures to neuropathic OP compounds that could arise through occupational, environmental, or accidental means, or from warfare or terrorist acts.

\section{Acknowledgements}

The research was supported by Project \#1055.2 of the International Science and Technology Center (ISTC), Projects \#RB2-2035 and \#RB2-2488 of the U.S. Civilian Research and Development Foundation (CRDF), and Grant \#DAAD19-021-0388 from the US Army Research Office.

\section{References}

Duckworth H, Coleman J (1970) Physicochemical and kinetic properties of mushroom tyrosinase. J. Biol. Chem. 245: 1613-1625.

Glynn P (1999) Neuropathy target esterase. Biochem. J. 344: 625-631.

Huggins DJ, Richardson RJ (1999) Brainstem axolemmal protein phosphorylation in vitro in hens dosed with di-1butyl-2,2-dichlorovinyl phosphate. J. Toxicol. Environ. Health, Part A 56: 263-282.

Johnson MK (1977) Improved assay of neurotoxic esterase for screening organophosphates for delayed neurotoxicity potential. Arch. Toxicol. 67: 113-115.

Johnson MK (1982) The target for initiation of delayed neurotoxicity by organophosphorus esters: biochemical studies and toxicological applications. In: Hodgson E, Bend JR, Philpot RM, eds. Reviews in Biochemical Toxicology, Amsterdam: Elsevier, 4 pp. 141-212.

Johnson MK (1990) Organophosphates and delayed neuropathy - is NTE alive and well? Toxicol. Appl. Pharmacol. 102: 385-399.

Kayyali US, Moore TB, Randall JC, Richardson RJ (1991) Neurotoxic esterase (NTE) assay: optimized conditions based on detergent-induced shifts in the phenol/4-aminoantipyrine chromophore spectrum. J. Anal. Toxicol. 15: 86-89.

Kotte H, Gründig B, Vorlop K-D, Strehlitz B, Stottmeister U (1995) Methylphenasonium-modified enzyme sensor based on polymer thick films for subnanomolar detection of phenols. Anal. Chem. 67: 65-70.

Lotti M (1986) Biological monitoring for organophosphateinduced delayed polyneuropathy. Toxicol. Lett. 33: 167-172. 
Makhaeva GF, Malygin VV (1999) A stable preparation of hen brain neuropathy target esterase for rapid biochemical assessment of neurotoxic potential of organophosphates. Chem.-Biol. Interact. 119/120: 551-557.

Makhaeva GF, Malygin VV, Martynov IV (2001) Assessment of neuropathic potential of some methyl- and phenylphosphonates using a stable preparation of neuropathy target esterase from chicken brain. Doklady Biochem. Biophys. 377: 68-71.

Makhaeva GF, Sigolaeva LV, Zhuravleva LV, Eremenko AV, Kurochkin IN, Richardson RJ, Malygin VV (2002) Neuropathy target esterase in whole blood: biomarker for exposure to neuropathic organophosphorus compounds. ASA Newslett. 02-05: 16-21.

Makhaeva GF, Sigolaeva LV, Zhuravleva LV, Eremenko AV, Kurochkin IN, Richardson RJ, Malygin VV (2003) Biosensor detection of neuropathy target esterase in whole blood as a biomarker of exposure to neuropathic organophosphorus compounds. J. Toxicol. Environ. Health, Part A 66: 599-610.

Malygin VV, Sokolov VB, Richardson RJ, Makhaeva GF (2003) Quantitative structure-activity relationships predict the delayed neurotoxicity potential of a series of O-alkyl-Omethylchloroformino phenylphosphonates. J. Toxicol. Environ. Health Part A 66: 611-625.

Richardson RJ (1995) Assessment of the neurotoxic potential of Chlorpyrifos relative to other organophosphorus com- pounds: a critical review of the literature. J. Toxicol. Environ. Health. 44: 135-165.

Richardson RJ (1998) Neurotoxicity, Delayed. In: Wexler P, (ed). Encyclopedia of Toxicology, Vol. 2. San Diego: Academic Press, pp. 385-389.

Richardson RJ, Davis CS, Johnson MK (1979) Subcellular distribution of marker enzymes and neurotoxic esterase in adult hen brain. J. Neurochem. 32: 607-615.

Shan D, Mousty C, Cosnier S, Shaolin M (2002) A composite poly azure B-clay-enzyme sensor for the mediated electrochemical determination of phenols. J. Electroanal. Chem. 537: 103-109.

Sigolaeva LV, Eremenko AV, Makower A, Makhaeva GF, Malygin VV, Kurochkin IN (1999) A new approach for determination of neuropathy target esterase activity. Chem.Biol. Interact. 119/120: 559-565.

Sigolaeva L, Makower A, Eremenko A, Makhaeva G, Malygin V, Kurochkin I, Scheller F (2001) Bioelectrochemical analysis of neuropathy target esterase in blood. Anal. Biochem. 290: $1-9$

Wang G, Xu J, Ye L, Zhu J, Chen H (2002) Highly sensitive sensors based on the immobilization of tyrosinase in chitosan. Bioelectrochemistry 57: 33-38. 